\title{
Citron Kinase Is Required for Postnatal Neurogenesis in the Hippocampus
}

\author{
James B. Ackman ${ }^{a}$ Raddy L. Ramos ${ }^{a}$ Matthew R. Sarkisian ${ }^{b}$ Joseph J. LoTurco ${ }^{a}$ \\ ${ }^{a}$ Department of Physiology and Neurobiology, University of Connecticut, Storrs, Conn., and \\ ${ }^{b}$ Department of Neurobiology, Yale University School of Medicine, New Haven, Conn., USA
}

\section{Key Words}

Neural stem cells · Neurogenesis · Dentate gyrus • Epilepsy

\begin{abstract}
The dentate gyrus is a site of continual neurogenesis in the postnatal mammalian brain. Here we investigated postnatal neurogenesis in the citron kinase (citron-K) null-mutant rat (flathead). The flathead rat has substantial deficits in embryonic neurogenesis that are due to failed cytokinesis and cell death. We report here the loss of citron-K function has an even severer effect on postnatal neurogenesis in the dentate gyrus. Analysis of phosphorylated histone H3 expression in postnatal neurogenic regions of the flathead mutant revealed a complete lack of mitotic cells in the dentate gyrus and a large reduction in the number of dividing cells in the flathead subventricular zone. Examination of 5-bromodeoxyuridine incorporation in the flathead rat revealed that the flathead rat had a $99 \%$ reduction in the number of newly generated cells in the dentate gyrus at postnatal day 10. In addition, doublecortin-positive cells were essentially absent from the postnatal flathead dentate gyrus which also lacked the vimentin-and nestin-positive radial glia scaffold that defines the neurogenic niche in the postnatal subgranular zone. Together these results indicate that postnatal neurogenesis in the dentate gyrus is eliminated by loss of citron- $K$ function, and suggests that a citron-K-dependent progenitor lineage forms the postnatal neuronal progenitor population in the dentate gyrus.

Copyright $\odot 2007$ S. Karger AG, Basel
\end{abstract}

\section{KARGER}

Fax +4161306 1234

E-Mail karger@karger.ch

www.karger.com (c) 2007 S. Karger AG, Basel

Accessible online at: www.karger.com/dne

\section{Introduction}

Neurogenesis is a continual process in the dentate gyrus of the hippocampus. Generation of dentate granule neurons has been shown to occur in the adult brain of rodents [Altman and Das, 1965; Cameron et al., 1993; Kaplan and Hinds, 1977], primates [Gould et al., 1999; Kornack and Rakic, 1999], and humans [Eriksson et al., 1998]. These newly born dentate granule neurons originate from a pool of neural stem cells present within the subgranular zone (SGZ) of the dentate gyrus [Gage et al., 1995; Palmer et al., 1997; Seri et al., 2001]. Neural stem cells present in neurogenic regions of the adult mammalian brain, including the SGZ and the subventricular zone (SVZ), have been identified as a constituent of the radial glia-astrocyte lineage [Doetsch et al., 1999a, b; Laywell et al., 2000; Imura et al., 2003; Merkle et al., 2004; Garcia et al., 2004; Seri et al., 2001].

Though neurogenesis occurs in the dentate gyrus throughout life, the peak of dentate granule cell production occurs during the first 2 weeks of postnatal development in rodents [Bayer, 1980; Schlessinger et al., 1975]. This period of peak neurogenesis occurs following a number of discrete developmental steps that take place during embryonic and postnatal development [Altman and Bayer, 1990a, b; Li and Pleasure, 2005]. Among the initial steps in dentate gyrus development is the migration of the earliest-born granule neurons from their place of birth in the neuroepithelium to the dentate gyrus anlage to form the primordial granule cell layer [Altman 
and Bayer, 1990a, b]. These pioneer granule cells migrate along the radial glia scaffold that extends from the ventricular zone (VZ) to the dentate primordium together with a secondary population of neurogenic progenitors [Altman and Bayer, 1990a, b; Rickmann et al., 1987]. This secondary progenitor population is established in the hilar region of the developing dentate formation during late embryonic development and undergoes a radial transformation to form the SGZ neuroepithelium from which postnatal and adult neurogenesis will originate [Altman and Bayer, 1990a, b; Eckenhoff and Rakic, 1984; Guéneau et al., 1982; Alvarez-Buylla and Lim, 2004].

The flathead mutant rat exhibits a central nervous system-specific phenotype that includes severe micrencephaly and frequent seizures [Sarkisian et al., 1999]. The numbers of neurons in the cerebellum, dentate gyrus, olfactory bulb, and upper neocortical layers are dramatically reduced in the flathead rat and the brain is 50\% smaller than that of the wild type at birth. Although migration in and lamination of the flathead neocortex are intact, up to $50 \%$ of pyramidal and nonpyramidal neurons are binucleate, indicating a cytokinesis failure during embryonic development [Sarkisian et al., 2001]. In addition, loss of citron kinase (citron-K) function causes deficits in mitosis [LoTurco et al., 2003] and an increase in apoptosis [Di Cunto et al., 2000; Roberts et al., 2000; Sarkisian et al., 2001]. The flathead mutation has been identified as a premature stop codon in the first exon of the citron-K gene [Sarkisian et al., 2002]. Citron- $K$ is expressed in dividing cells during embryonic development where it localizes to the cleavage furrow of cells undergoing cytokinesis but is absent in the flathead mutant [Sarkisian et al., 2002; Di Cunto et al., 2000]. It is hypothesized that citron- $\mathrm{K}$ is essential for cytokinesis in neurogenic divisions, since citron- $\mathrm{K}$ is expressed in the developing cerebral cortex during the peak period for generation of late-born cortical neurons, and because many neurons in the flathead mutant are binucleate whereas glia are not binucleate [LoTurco et al., 2003].

Here, we show that there is a dramatic reduction in the number of neurons in the dentate gyrus of the flathead rat, greater than the decrease in the brain at large. The decrease is associated with a near loss of dividing cells and new neurons in the postnatal dentate germinal zone (DGZ). In comparison, there is a more modest but significant reduction in the numbers of dividing cells and neuroblasts in the postnatal SVZ and rostral migratory stream (RMS) of the flathead rat. These results demonstrate that postnatal neurogenesis is limited in the SVZ/RMS and virtually absent in the dentate gyrus after loss of citron-K function.

\section{Materials and Methods}

Animals and 5-Bromodeoxyuridine Injections

All animal procedures were performed according to protocols approved by the University of Connecticut Animal Care and Use Committee. Flathead mutant rats were obtained from bred colonies at the University of Connecticut. For all experiments, normal littermates from flathead litters were used as controls and were designated as wild type. The normal littermates included both heterozygous and homozygous wild-type animals. Brains from flathead and littermate rats were examined at embryonic day 14 (E14), postnatal day 0 (P0), P7, P14, P16, P17, P19, P21, P22, and P24.

As for 5-bromodeoxyuridine (BrdU) injections, pregnant dams received $50 \mathrm{mg} / \mathrm{kg}$ BrdU intraperitoneally at 14 and 15 days of gestation. Postnatal flathead and control pups received $40 \mathrm{mg} /$ $\mathrm{kg}$ BrdU intraperitoneally. Embryonic rat brains were collected into cold HBSS and fixed overnight in $4 \%$ paraformaldehyde (PFA). Postnatal rat pups were euthanized with wet ice (less than P7) or isoflurane (greater than P7) and perfused intracardially with phosphate-buffered saline (PBS) followed by $4 \%$ PFA. Brains were dissected out and postfixed overnight at $4^{\circ} \mathrm{C}$ in $4 \%$ PFA followed by embedding in $2 \%$ agarose. They were cut at $40 \mu \mathrm{m}$ on a vibratome (Leica VT-1000S). Free-floating sections were stored at $-20^{\circ} \mathrm{C}$ in a cryoprotectant solution (PVP- $40,30 \%$ sucrose, in ethylene glycol and $0.2 \mathrm{M}$ phosphate buffer, $\mathrm{pH}$ 7.4) until use.

\section{Immunocytochemistry, Histochemistry, and Confocal}

Microscopy

BrdU immunocytochemistry was performed by rinsing freefloating brain sections in PBS followed by denaturation in $2 \mathrm{~N} \mathrm{HCl}$ for $30 \mathrm{~min}$ at room temperature. Sections were neutralized in $0.1 \%$ sodium borate and rinsed in PBS before continuation with the standard immunocytochemistry protocol. The standard immunocytochemistry protocol included blocking sections in 5\% donkey serum (Sigma, No. D9663) + 0.3\% Triton X-100 for $1 \mathrm{~h}$ at room temperature followed by incubation in primary antibodies overnight at $4^{\circ} \mathrm{C}$. After rinsing for $1 \mathrm{~h}$, sections were incubated in secondary antibodies for $1 \mathrm{~h}$ at room temperature followed by a final rinse in PBS, mounted on glass slides, and coverslipped in Prolong Antifade (Molecular Probes). The primary antibodies used were: rat anti-BrdU (1:100, Accurate, No. OBT0030), goat antidoublecortin (1:100, Santa Cruz, No. sc-8066), mouse anti-NeuN (1:1,000, Chemicon, No. MAB377), mouse anticalbindin D28K (1:1,000, Sigma, No. C8666), rabbit antiphospho-histone H3 (1:500, Chemicon, No. 06-570), mouse antivimentin (1:200, Chemicon, No. MAB3400), mouse antinestin (1:200, Chemicon, No. MAB353), and mouse anti-GFAP (1:200, Sigma, No. G3893). The secondary antibodies used were: donkey antirabbit Alexa 488 (1:200, Molecular Probes), donkey antimouse Alexa 488 (1:200, Molecular Probes), donkey antirat Cy3 (1:200, Jackson ImmunoResearch), donkey antimouse Alexa 568 (1:200, Molecular Probes), and donkey antimouse Cy5 (1:200, Jackson ImmunoResearch). Some sections were counterstained with the nuclear label TO-PRO-3 (Molecular Probes).

For use with nonfluorescent histochemistry, sections were rinsed several times with $2.5 \%$ normal goat serum in PBS and then incubated in biotinylated secondary antibodies (biotinylated goat antimouse; 1:200, Vector Labs, Burlingame, Calif., USA) for $2 \mathrm{~h}$ at room temperature. Sections were rinsed several times with 
PBS and incubated for $1 \mathrm{~h}$ in an avidin-horseradish peroxidase mixture. Then they were rinsed in PBS and reacted with $0.05 \%$ diaminobenzidine in the presence of $0.0015 \% \mathrm{H}_{2} \mathrm{O}_{2}$. Sections were collected onto gelatin-coated slides, dried for several hours, and coverslipped with Cytoseal.

For Timm histochemistry, rats were perfused with a sodium

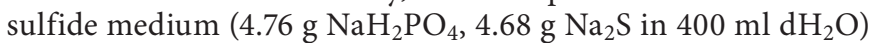
followed by $4 \%$ PFA. Brains were cryoprotected in $30 \%$ sucrose and frozen over liquid $\mathrm{N}_{2}$. Sections $(20 \mu \mathrm{m})$ were developed in the dark for 30-60 min in a solution containing gum arabic $(120 \mathrm{ml}$ of a $20 \%$ stock), citrate buffer ( $20 \mathrm{ml}$ of a $2 \mathrm{M}$ stock), hydroquinone ( $60 \mathrm{ml}$ of a $5.7 \%$ stock), and $0.17 \mathrm{~g}$ silver nitrate. Sections were dehydrated in ethanol, cleared in xylene and coverslipped with DPX.

Slides were imaged with either a Nikon E400 microscope and Spot camera or with a laser scanning confocal microscope (Leica TCS-SP2; laser lines at 488, 543, 633). Confocal images were acquired at $0.3-1.0 \mu \mathrm{m}$ per $\mathrm{z}$-section.

\section{Quantification}

For quantification of mitotic cells, the number of phosphorylated histone $\mathrm{H} 3$ (phosH3)-positive cells per sagittal section was counted for the dentate gyrus and the SVZ including the first $300 \mu \mathrm{m}$ of the RMS from the flathead rats (ages P10, P16, and P22) and the wild-type rats (ages P16 and P22). For BrdU quantification, animals aged P17 or P22 that had received BrdU injections at $\mathrm{P} 10$ or P19, respectively, were used. In the dentate gyrus, the number of BrdU-positive cells per sagittal section was counted. For the SVZ, confocal images (8-bit indexed color) from sagittal sections were thresholded in Image J with a tolerance of 21 and the polygon selection tool was used to outline the SVZ and the first $300 \mu \mathrm{m}$ of the RMS. The analyze particles tool with a pixel radius of 3 was used to automatically count the number of BrdUpositive cells in the SVZ. Several sections of the flathead and littermate SVZ were hand counted to confirm the validity of the automatic counting procedure. For both phosH3 and BrdU quantification in the dentate gyrus and SVZ, the mean and SEM for the number of positive cells per section were calculated for the flathead rat and wild type. For all conditions, quantification was performed on an average of 13 sagittal sections from the medial extent (range of 0.5-2.5 mm from midline). Differences between compared means were determined with Student's $t$ test, with $\mathrm{p}<$ 0.05 considered significant (for all experiments: $\mathrm{N}=$ number of animals, $\mathrm{n}=$ number of stained sections).

\section{Results}

\section{Granule Layer Dysgenesis in the flathead Dentate} Gyrus

The brain of the citron-K null mutant (flathead) is approximately one-half the size of that of the wild type at birth. Areas of the central nervous system that undergo significant amounts of late embryonic or postnatal neurogenesis, such as the retina, cerebellum, olfactory bulb, and dentate gyrus, are the regions most affected by a lack of citron-K function [Di Cunto et al., 2000; Roberts et al.,
2000]. The dentate gyrus is markedly reduced in size in the flathead rat (fig. 1a-c). Nissl-stained sagittal and coronal sections of P21 wild-type and flathead brains reveal that the inferior blade of the dentate gyrus is missing in the flathead rat (fig. 1b, c). The superior blade of the dentate gyrus that is present in the flathead rat is severely reduced in the dorsoventral and mediolateral dimensions. In addition, the flathead dentate gyrus is reduced in the rostrocaudal extent. In contrast to the dentate gyrus, the CA3-CA1 pyramidal layers of Ammon's horn are more moderately reduced in size in the flathead rat and have no apparent gross disruption in organization. To confirm that the cells remaining in the disrupted granule layer of the flathead dentate gyrus were granule neurons, we performed calbindin D28K immunocytochemistry on horizontal sections of P21 flathead and wild-type hippocampi (fig. 1e). We found that calbindin was expressed by cells in the granule cell layer of the flathead rat. Furthermore, we performed Timm's silver sulfide staining on horizontal sections of P14 wild-type and flathead hippocampi (fig. 1d). We found that Timm staining intensely labeled granule cell mossy fiber axons in the hilar region and CA3 pyramidal layer of the flathead hippocampus in a pattern appropriate for mature dentate granule neurons.

\section{Loss of Progenitor Cells in the Postnatal flathead \\ Dentate Gyrus}

Next we determined whether proliferating cells were present in the SGZ of the flathead dentate gyrus using phosH3 and BrdU immunocytochemistry (fig. 2). There was a lack of phosH3-positive dividing cells in the flathead dentate gyrus at P16 and P22 (fig. 2a, b, f). The number of phosH3-positive cells per section in the flathead rat was reduced by $97 \%$ with respect to the wild type $(0.3 \pm$ $0.1, \mathrm{~N}=4, \mathrm{n}=19$ for the flathead rat; $8.8 \pm 2.9, \mathrm{~N}=2$, $\mathrm{n}=5$ for the wild type; $\mathrm{p}<0.05)$. Consistent with a lack of proliferating cells in the dentate gyrus, the number of cells in the P17 flathead dentate gyrus that incorporated BrdU at $\mathrm{P} 10$ was reduced by $99 \%$ (fig. 2 c, d, g; $3.5 \pm 0.9$ cells/ section, $\mathrm{N}=7, \mathrm{n}=11$ for the flathead rat; $364.3 \pm 32.0$ cells/section, $\mathrm{N}=7, \mathrm{n}=18$ for the wild type; $\mathrm{p}<0.001$ ).

Next we tested if dentate granule neurons in the flathead rat were generated during embryonic development. Consistent with an embryonic birth date, we found that granule neurons in the P19 flathead dentate gyrus were labelled by BrdU administered at E14 and E15 (fig. 2e; $\mathrm{N}=1$ ). Note the presence of several binucleate NeuNpositive cells near the internal limb of the dentate gyrus (arrowheads, fig. 2e). 
a

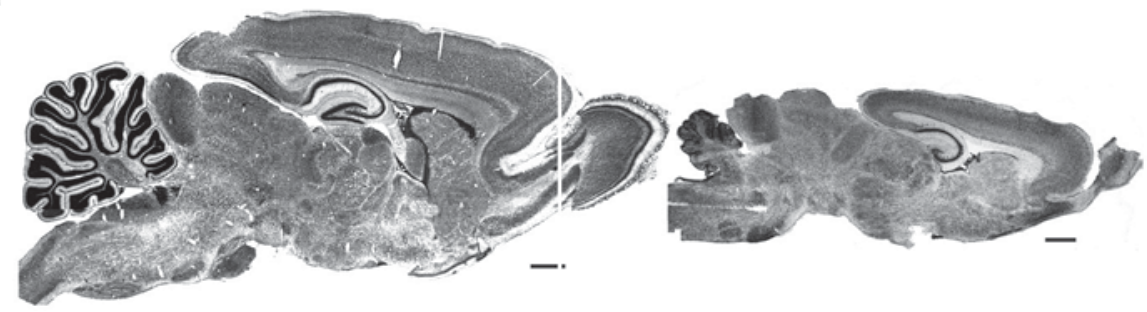

b

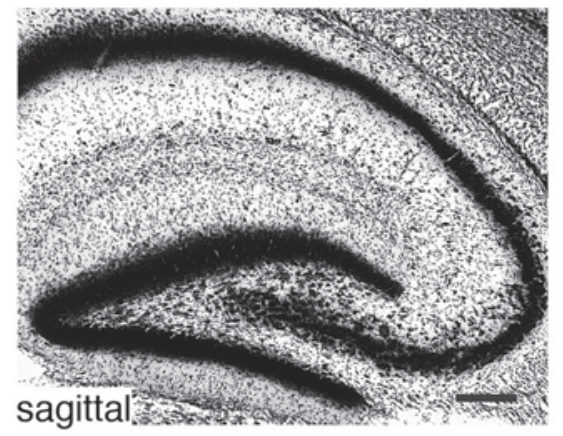

c

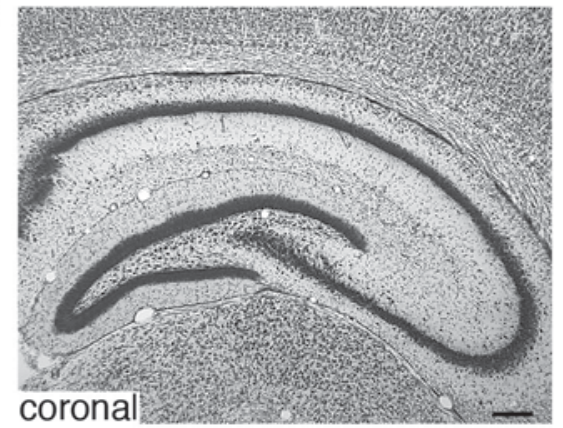

d

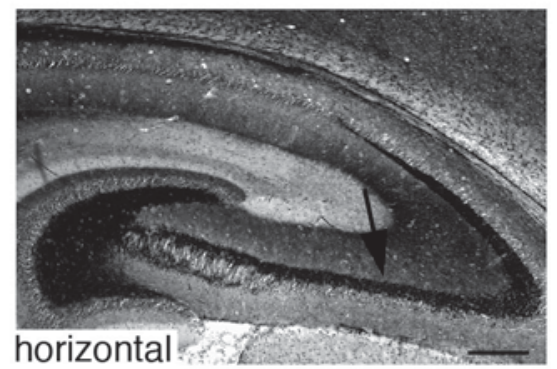

sagittal sections of the wild-type and flat head hippocampus. c Higher-magnification comparison of Nissl staining in coronal sections of the P21 wild-type and flathead hippocampus. $\mathbf{d}$ Timm staining of granule cell mossy fiber axons (arrows) in horizontal sections of the P14 wild-type and flathead hippocampus. e Expression of calbindin in horizontal sections of the P21 wild-type and flathead dentate gyrus. In the images, medial is down and rostral is to the right. Scale bars: $1 \mathrm{~mm}(\mathbf{a}) ; 250 \mu \mathrm{m}$ (b-e).

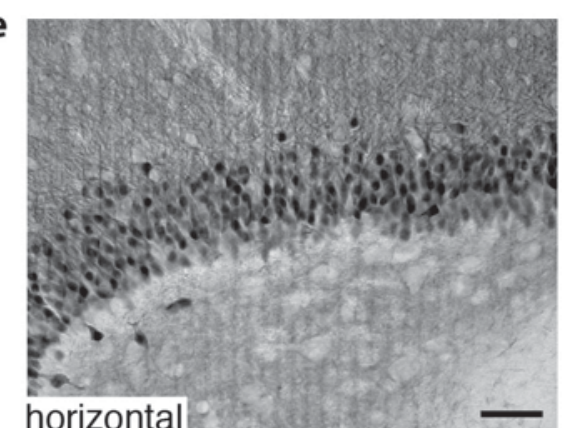

Fig. 1. Dysgenesis of the granule cell layer in the flathead dentate gyrus. a Nisslstained sagittal sections of wild-type and flathead brains at P21. b Higher-magnification comparison of Nissl staining in flathead rat
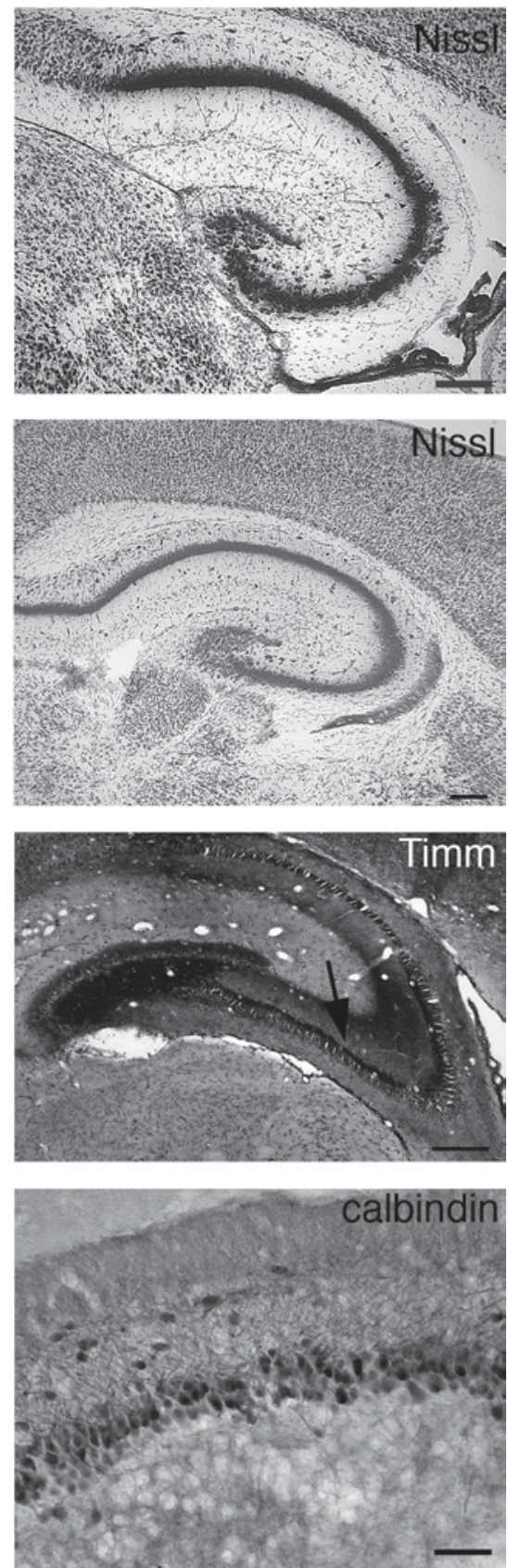
Decreased Proliferation in the SVZ and RMS of the flathead Rat

Since the SVZ/RMS is a postnatal neurogenic region, we tested for expression of the immature neuron marker, doublecortin, in the SVZ/RMS of the flathead rat. Doublecortin expression was reduced in the SVZ and RMS of the flathead rat at P22 (fig. 3a). Next we determined whether there was a decrease in the numbers of mitotic progenitors in the SVZ/RMS of the flathead rat. Analysis of phosH3 immunocytochemistry on sagittal sections of the P22 flathead brain revealed that the number of phosH3-expressing cells per section was reduced by $92 \%$ in the flathead SVZ/RMS (fig. 3b, d; $11.8 \pm 1.7$ cells/section, $\mathrm{N}=4, \mathrm{n}=19$ for the flathead rat; $146.8 \pm 26.9$ cells/ section, $\mathrm{N}=2, \mathrm{n}=4$ for the wild type; $\mathrm{p}<0.05)$. BrdU immunocytochemistry on sections from P17 animals that received BrdU at $\mathrm{P} 10$ confirmed a reduction in newly generated cells in the flathead SVZ/RMS (fig. 3c, e). The decrease in BrdU-labeled cells per section was 83\% in the flathead SVZ/RMS (179.4 \pm 39.8 cells/section, $\mathrm{N}=5, \mathrm{n}=15$ for the flathead rat; $1,059.7 \pm 113.2$ cells/ section, $\mathrm{N}=4, \mathrm{n}=9$ for the wild type; $\mathrm{p}<0.001$ ).

\section{Altered Neurogenic Niche in the flathead Dentate \\ Gyrus}

Next we assessed whether doublecortin-expressing cells were present in the postnatal flathead dentate gyrus. In contrast to the wild type where there was doublecortin immunoreactivity throughout the dentate gyrus at P17 and P22, most immunostained sections from the flathead rat contained no doublecortin-positive cells or fibers (fig. $4 \mathrm{a}, \mathrm{b} ; 0.39 \pm 0.29$ cells/section, $\mathrm{N}=8, \mathrm{n}=23$ for the flathead rat; $\mathrm{N}=3, \mathrm{n}=8$ for the wild type). In the few sections of the flathead hippocampus where isolated doublecortin immunoreactivity was present, the positive cells were located in the hilar region between the dentate

Fig. 2. Proliferating cells are absent in the postnatal flathead dentate gyrus. a Lack of phosH3-positive nuclei (white) in the flathead SGZ at P16. The section is counterstained with the nuclear label TO-PRO-3 (blue). b Lack of phosH3-positive nuclei in the flathead SGZ at P22. Dashed lines outline the granule cell layer. c Decreased numbers of BrdU-positive cells in the postnatal flathead dentate gyrus. The images are from P17 wild-type and flathead animals that received BrdU injections at P10. The few BrdUpositive objects next to the dentate gyrus in this image are blood vessels. $\mathbf{d}$ Lack of newly generated granule neurons in the flathead dentate gyrus. BrdU is green and NeuN is red. e Dentate granule neurons in the flathead rat incorporate BrdU at E14-E15 (arrows). Image is from a P19 flathead rat that received BrdU on E14 and E15. Binucleate neurons are indicated by arrowheads. f Quantifi-
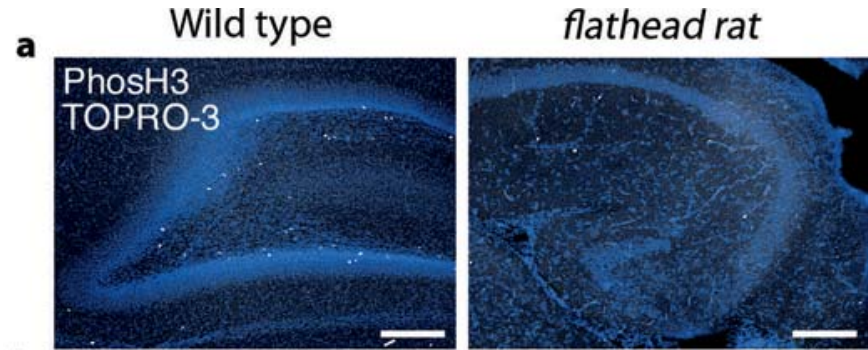

b
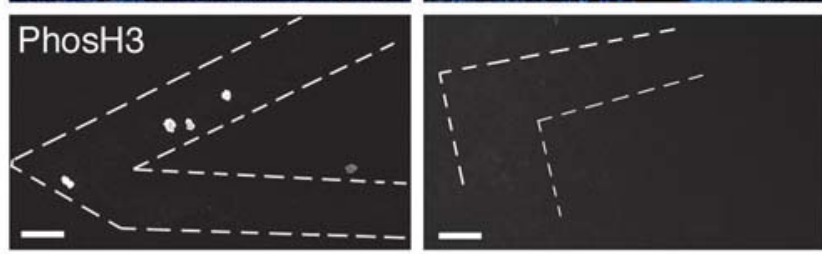

C
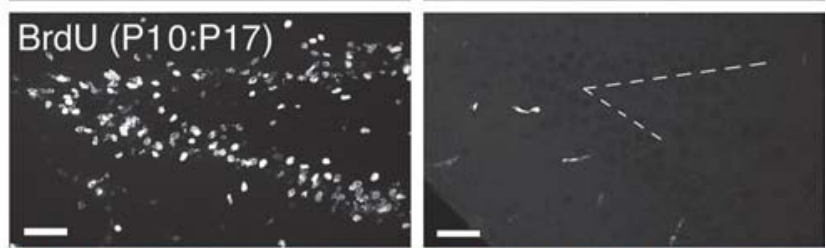

d
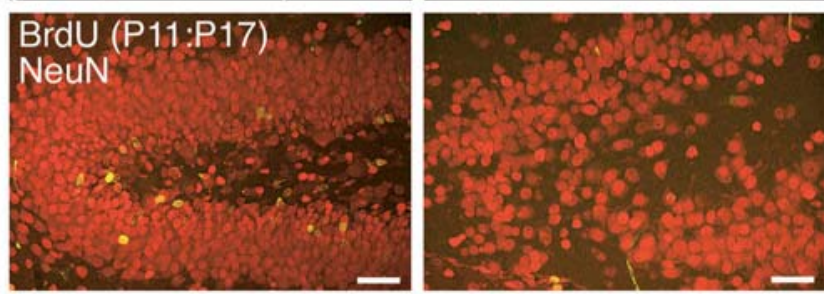


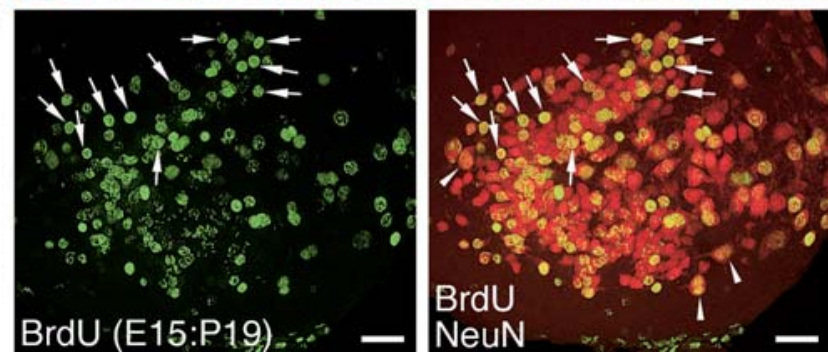

f
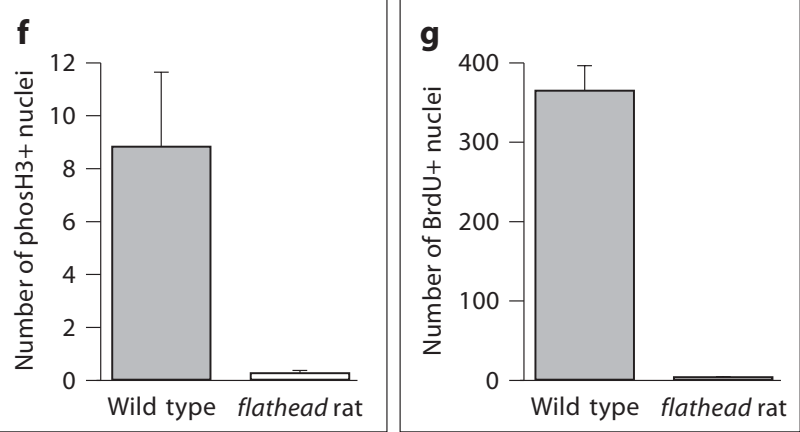

cation of phosH3-positive nuclei in the SGZ. Numbers are expressed as the mean number of phosH3-positive cells in the SGZ per section $(\mathrm{p}<0.05)$. g Quantification of the number of BrdUpositive cells in the dentate gyrus. Numbers are expressed as the mean number of BrdU-positive nuclei per section $(\mathrm{p}<0.001)$. Scale bars: $40 \mu \mathrm{m}$ (a-e). 

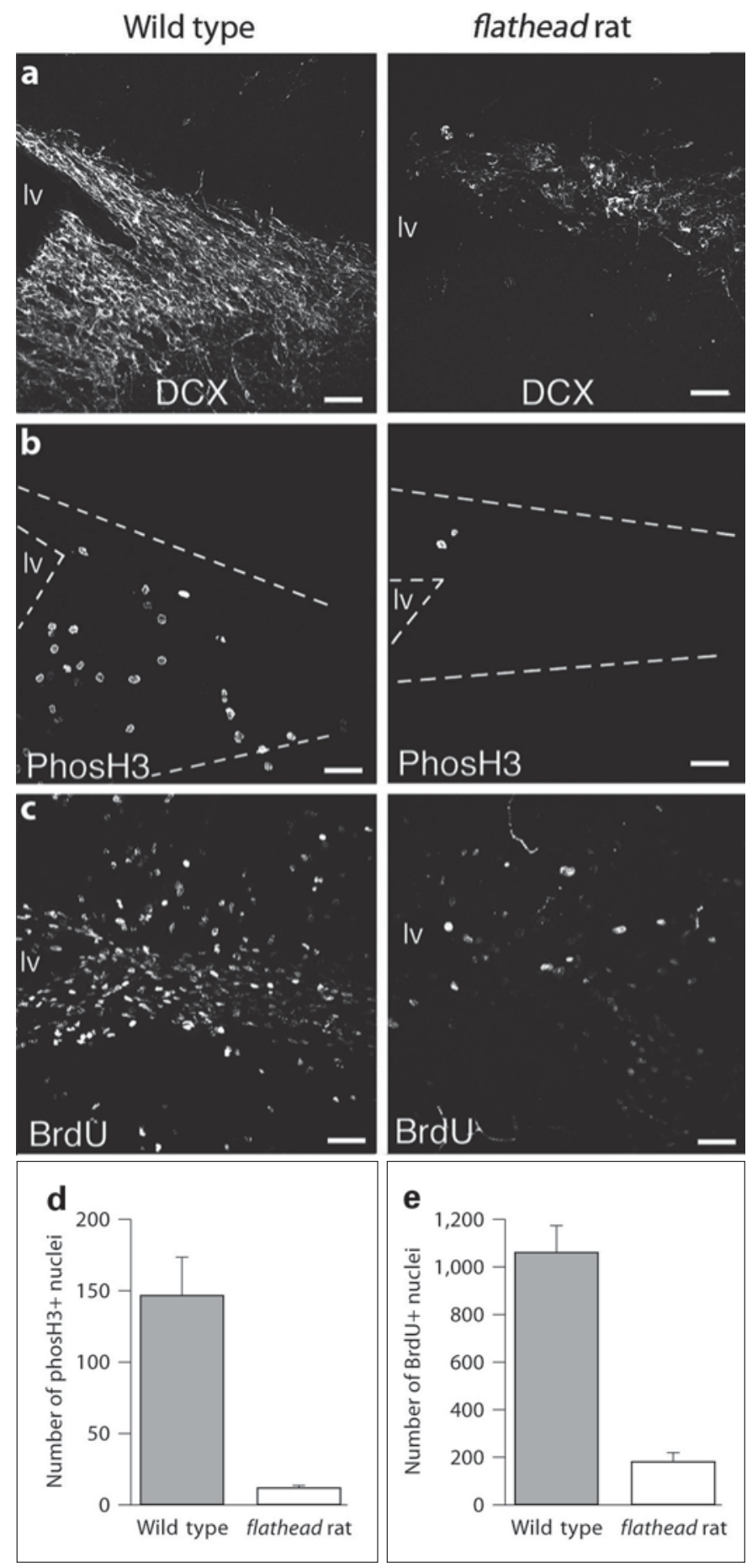

Fig. 3. Decreased proliferation in the postnatal SVZ of the flathead rat. a Expression of doublecortin (DCX) and phosH3 in the SVZ of the wild-type and flathead rat at P22. b Reduced numbers of phosH3-positive nuclei in the flathead SVZ at P22. The images are from the same sections shown in a. c Decreased numbers of BrdU-positive cells in the postnatal flathead SVZ. The images are from P17 wild-type and flathead animals that received BrdU injections at P10. d Quantification of phosH3-positive nuclei in the horn and CA3 (fig. 4b), whereas doublecortin-positive cells were never found outside the granule cell layer or SGZ in the wild-type dentate gyrus. In sagittal stained sections, the dendrites of hilar doublecortin-positive cells in the flathead rat were generally oriented in the caudal or ventral directions towards the dentate molecular layer.

The postnatal SGZ of the dentate gyrus contains radial-type astrocytes that originate from radial glia during development [Eckenhoff and Rakic, 1984; Rickmann et al., 1987]. Radial-type astrocytes generate granule neurons in the dentate gyrus [Seri et al., 2004] and express the intermediate filament proteins vimentin, nestin, and GFAP [Eckenhoff and Rakic, 1984; Rickmann et al., 1987; Lendahl et al., 1990; Seri et al., 2004]. To determine whether radial-type astrocytes were present in the postnatal flathead SGZ, we performed vimentin, nestin, and GFAP immunocytochemistry on sagittal sections of the wild-type and flathead dentate gyrus at P21-P24. The postnatal flathead dentate gyrus lacked vimentin- and nestin-positive radial fibers (fig. $4 \mathrm{c}, \mathrm{d}$ ) and contained few GFAP-positive radial fibers (arrows in fig. 4e). In contrast to the reduction in radial-type astrocytes, multipolar astrocytes that expressed GFAP were abundant in the flathead SGZ, as well as in the hilus and in the molecular layer. In other postnatal neurogenic regions, such as the SVZ/RMS, both radial glia and multipolar-type astrocytes that had intense staining for vimentin, nestin, and GFAP were present in the flathead rat (data not shown).

\section{Reduced Migration of Dentate Precursors during \\ Development of the flathead Dentate Gyrus}

Next we examined the cytoarchitecture of germinative matrices associated with the development of the dentate gyrus. The hippocampal neuroepithelium is established along the medial wall of the lateral ventricle during embryonic development (fig. 5a). An indentation of the hippocampal neuroepithelium, the dentate notch, is the location of the primary dentate neuroepithelium from which the earliest-generated granule cells originate as well as migrating progenitors that establish the secondary dentate

SVZ. Numbers are expressed as the mean number of phosH3positive cells in the SVZ per section $(\mathrm{p}<0.05)$. e Quantification of the number of newly generated cells in the SVZ. Numbers are expressed as the mean number of BrdU-positive nuclei in the SVZ $(\mathrm{p}<0.001)$. Scale bars: $40 \mu \mathrm{m}(\mathbf{a}-\mathbf{c})$. 
matrix. We analyzed Nissl-stained coronal sections of the hippocampal neuroepithelium at E14 to see if any gross cytoarchitectural abnormalities were present in the primary dentate neuroepithelium of the flathead rat before formation of the secondary dentate matrix. No distinct alterations were present in the primary dentate neuroepithelium of the flathead rat at E14 (fig. 5b). The secondary dentate matrix is a germinative matrix located adjacent to the $\mathrm{VZ}$ of the dentate neuroepithelium and becomes evident around E18 in the rat [Altman and Bayer, 1990a]. The secondary dentate matrix consists of proliferative cells and migratory cells and extends tangentially towards the dentate gyrus as a subpial migration. This dentate migration is robust during late embryonic and early postnatal development (E20-P5 in the rat) [Altman and Bayer, $1990 \mathrm{a}, \mathrm{b}]$ and contains neuroblasts that migrate to the granule cell layer and progenitors that migrate to establish the proliferative tertiary dentate matrix in the hilus (fig. 5c). We examined the dentate migration in the perinatal hippocampus, a time during which there are large numbers of cells migrating towards the dentate gyrus. Nissl-stained horizontal sections of the P0 flathead and wild-type hippocampus revealed that the dentate migration in the flathead rat is distinctly reduced (fig. 5d). In addition, there appeared to be a lack of cells aggregating in the region of the internal dentate blade in the flathead rat, as well as an areal reduction in the region of the tertiary dentate matrix. In contrast to the dentate migration, which appeared thin between the pial surface and CA3 (boxed area in fig. 5d), the SVZ, Ammon's horn, stratum radiatum, and the fimbria did not appear particularly reduced in the flathead rat at P0. Additionally, we observed an increased number of pyknotic nuclei in Nissl-stained sections at $\mathrm{P} 0$ in the secondary dentate matrix, the dentate migration, and the tertiary matrix in the flathead rat (data not shown). Increased cell death in the dentate germinative matrices is consistent with previous studies indicating that a lack of citron-K function leads to failed cytoki-

Fig. 4. Altered neurogenic niche in the flathead dentate gyrus. a Doublecortin (DCX) expression in the wild-type and flathead dentate gyrus at P17. Notice the dramatic reduction in doublecortin-positive cells and processes within the dentate granule cell layer of the flathead rat. $\mathbf{b}$ Doublecortin expression in the dentate gyrus of the wild-type and flathead rat at P22. c Vimentin expression in the dentate gyrus of the wild-type and flathead rat at P22. Notice the high density of vimentin-positive radial fibers in the wild-type dentate gyrus and the lack of radial fibers in the flathead rat. $\mathbf{d}$ Nestin expression in the dentate gyrus of the wild-type and flathead rat at P24. Nestin expression was located in endothe-
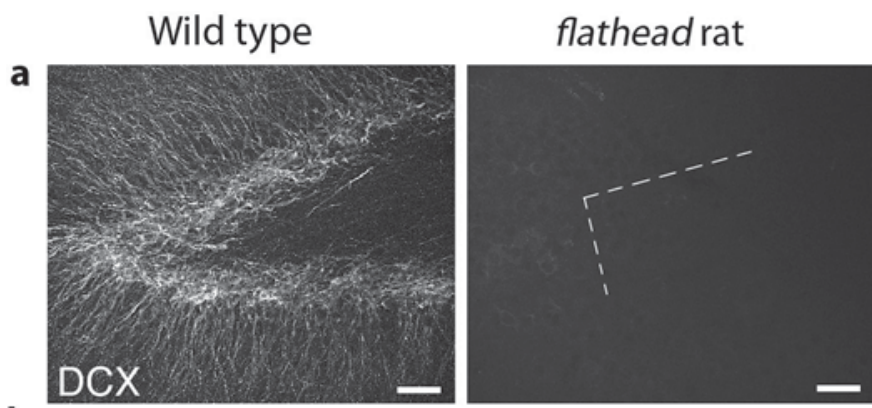

b
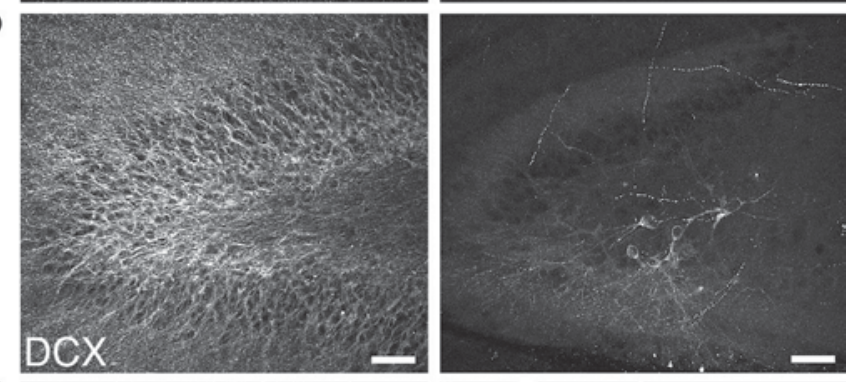

c
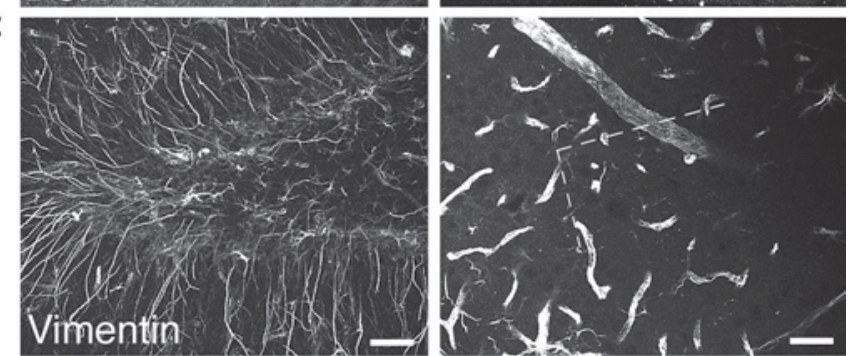

d
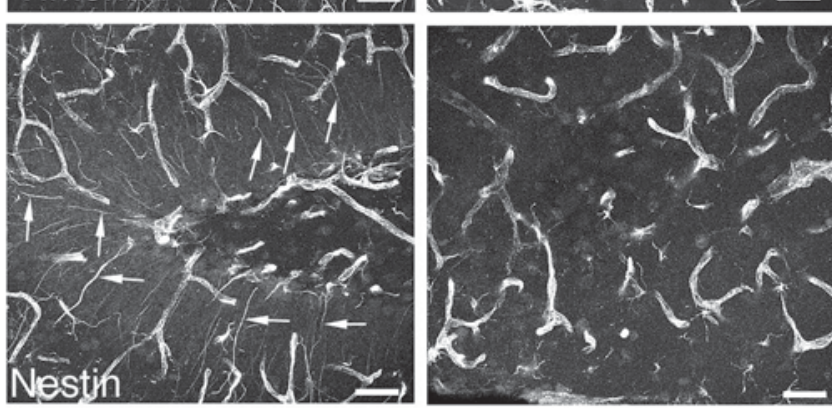

e
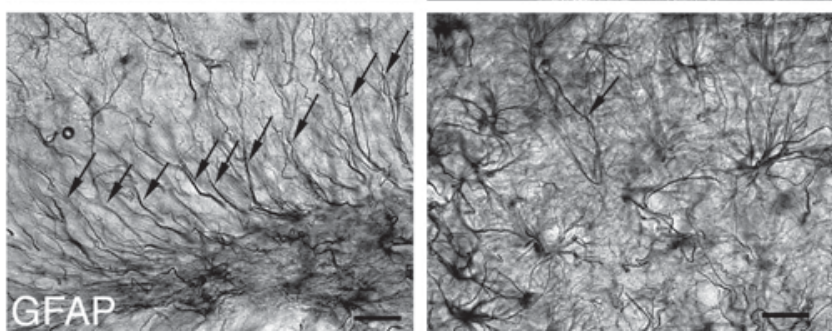

lial cells, multipolar astrocytes, and radial-type astrocytes in the wild-type dentate gyrus, whereas in the flathead dentate gyrus only nestin-positive endothelial cells and multipolar astrocytes were found. Arrows indicate examples of nestin-positive radial fibers in the wild-type dentate gyrus. e GFAP immunohistochemistry in the dentate gyrus of the wild-type and flathead rat at P21. Notice the lack of GFAP-positive radial fibers in the flathead dentate gyrus. Arrows indicate GFAP-positive radial fibers. Scale bars: $40 \mu \mathrm{m}(\mathbf{a}-\mathbf{d}) ; 25 \mu \mathrm{m}(\mathbf{e})$. 


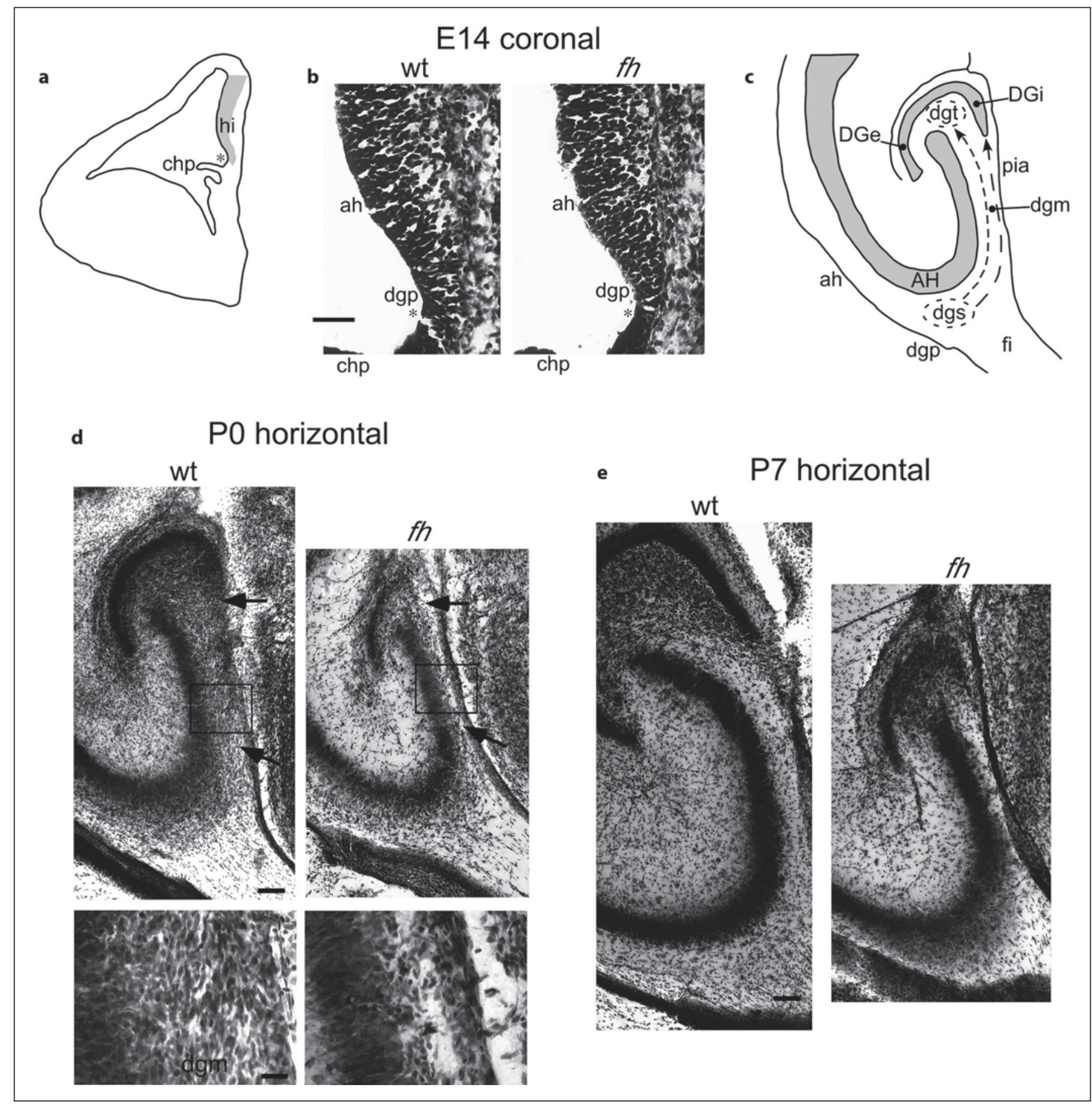

Fig. 5. Diminished dentate migratory stream during development of the flathead dentate gyrus. a Location of the hippocampal neuroepithelium (hi) in the developing rat brain. Dentate notch is indicated by an asterisk. chp $=$ Choroid plexus. $\mathbf{b}$ Nissl-stained coronal sections of the wild-type (wt) and flathead ( $f h$ ) hippocampal neuroepithelium at E14. Note the similarity in appearance between the wild-type and flathead ammonic neuroepithelium (ah) and primary dentate neuroepithelium (dgp). chp = Choroid plexus. c Overview of dentate gyrus development during the late embryonic and early postnatal periods. The dentate migratory stream (dgm) consists of neuroblasts and progenitors which migrate to form the internal blade of the dentate gyrus (DGi) and the proliferative tertiary dentate matrix (dgt) in the hilus. $\mathrm{AH}=\mathrm{Am}-$ mon's horn; DGe = external blade of the dentate gyrus; fi = fimbria; $\mathrm{dgs}=$ secondary dentate matrix; $\mathrm{ah}=$ ammonic neuroepithelium; dgp = primary dentate neuroepithelium. d Nissl-stained horizontal sections of the wild-type (wt) and flathead $(f h)$ hippocampus at P0. Note the decreased number of cells in the flathead dentate migratory stream (dgm) compared with the wild-type dentate migratory stream (arrows). The panel of high-magnifica- 
nesis and massive apoptosis in germinal zones such as the ganglionic eminence, neocortical VZ/SVZ, and the cerebellar granule layer [Roberts et al., 2000; Sarkisian et al., 2001; Di Cunto et al., 2000]. By P7, the density of cells in the dentate migration has decreased in both the wild-type and flathead rats (fig. 5e). Notice that by P7 the dentate gyrus has greatly increased in size and the internal blade of the dentate gyrus has formed in the wild type, whereas in the flathead rat, the dentate gyrus has not undergone a similar increase in size and the internal blade is mostly absent.

\section{Discussion}

Previous studies have demonstrated severe deficits in embyronic neurogenesis and increased apoptosis in citronK null mutants [Di Cunto et al., 2000; Roberts et al., 2000; Sarkisian et al., 2002]. Here, we show that postnatal neurogenesis is depleted in the citron-K null-mutant rat (flathead). The depletion of postnatal neurogenesis in the flathead rat can most likely be attributed to a loss of mitotic progenitors in postnatal neurogenic regions as proliferation was decreased in the SVZ/RMS and nearly completely absent in the dentate gyrus. These results indicate that citron-K function is required for development of the neurogenic progenitor pool in the postnatal dentate gyrus.

Among the developmental steps required for formation of the dentate gyrus is the establishment of a tertiary progenitor population in the SGZ during late embryonic and early postnatal development (also termed the 'tertiary germinative matrix'; see Altman and Bayer [1990a, b]). It seems likely from our results that the tertiary precursor pool fails to be established in the dentate gyrus of the citron-K null mutant (flathead) as there was a lack of mitotic progenitors in the SGZ, as well as a lack of newly generated neurons in the granule cell layer of the postnatal flathead hippocampus. In addition, radial-type astrocytes were absent in the postnatal flathead SGZ. Since radial-type astrocytes serve as progenitors of new granule neurons in the adult dentate gyrus [Seri et al., 2004], these results indicate that formation of the neurogenic niche is disrupted in the flathead SGZ.

tion images of the dentate migratory stream below were taken from the region outlined by the boxes in the upper panel. e Nisslstained horizontal sections of the wild-type (wt) and flathead ( $f h$ ) hippocampus at P7. Scale bars: $50 \mu \mathrm{m}$ (b); $100 \mu \mathrm{m}$ (d, upper panel; e); $25 \mu \mathrm{m}$ (d, lower panel).

Citron Kinase and Postnatal

Neurogenesis
Though there was a lack of neurogenesis in the postnatal flathead dentate gyrus, granule cells were present in the rudimentary granule layer of the flathead rat. These flathead granule cells possessed key differentiated granule neuron characteristics such as calbindin expression and Timm staining of mossy fiber axons. In addition, granule cells that were present in the flathead dentate gyrus were found to incorporate BrdU administered at E14E15, a time during which only the primordial granule neuron population is being generated. The presence of a rudimentary granule cell layer consisting of early-born granule neurons in the flathead rat indicates that the disruption in dentate gyrus development is fundamentally different from other rodent models involving deficits in SGZ precursor pool establishment such as Wnt signaling and CXCR4 mutants where the dentate gyrus completely fails to form [Galceran et al., 2000; Lee et al., 2000; Zhou et al., 2004; Bagri et al., 2002; Lu et al., 2002]. These results indicate that early in development generation of the pioneer granule neurons and migration into the dentate anlage can occur in the absence of citron-K function but that the subsequent progenitor-progenitor or progenitorneuron divisions from which the majority of granule neurons originates require citron-K function.

Exactly how citron-K function is involved in the development of the SGZ progenitor population is notknown. During embyronic development, citron- $K$ is expressed in a portion of dividing progenitors along the VZ surface resulting in about half of cortical neurons being multinucleate due to cytokinesis failure [Sarkisian et al., 2002]. The study of the postnatal brain with in situ hybridization has revealed that the citron- $\mathrm{K}$ message is expressed in the external granule layer of the cerebellum as well as in the SVZ and RMS of the forebrain [Di Cunto et al., 2000]. Although citron-K expression has not been reported in the postnatal dentate gyrus, it is possible that it is present at low levels and that a lack of citron-K function leads to progenitor cell depletion in the SGZ due to cytokinesis failure and apoptosis. Alternatively, a lack of citron-K function could cause progenitor cell depletion in the hippocampal neuroepithelium, before the tertiary precursor population migrates into the hilus and SGZ. Our results showing a diminished dentate migration from the secondary germinative matrix to the dentate formation indicate that a lack of citron- $\mathrm{K}$ function may deplete progenitors and neuroblasts before their migration into the hilus and the granule cell layer.

Citron-K mutants exhibit frequent seizures that begin near the end of the first postnatal week [Sarkisian et al., 1999; Di Cunto et al., 2000]. Temporal lobe epilepsy is 
often associated with disorganization or loss of cells in the granule cell layer of the dentate gyrus [Houser, 1990, 1992]. It seems possible that frequent seizure activity may have a detrimental effect on development of the flathead dentate gyrus. However, it seems unlikely that the frequent seizures in the flathead rat would cause the loss of the SGZ progenitor population. First, seizures in the flathead rat start at the end of the first postnatal week, after the secondary progenitor population has migrated to and become established in the SGZ/hilus [Sarkisian et al., 1999]. Second, seizure activity has been reported to increase granule neuron neurogenesis in the dentate gyrus [Parent et al., 1997]. Although the results presented here did not include analysis of BrdU incorporation in the postnatal flathead brain earlier than the first postnatal week, we have examined BrdU incorporation at P2 in flathead rats and found that the lack of dentate granule neuron generation was present at this early postnatal age as well (data not shown). We have not assessed proliferation at this early postnatal age with mitotic markers or short survival BrdU assays, but examination of the neonatal flathead hippocampus with Nissl staining indicates that the morphological abnormalities in dentate gyrus development are present in the flathead rat within the first postnatal week. In conclusion, postnatal neurogenesis in the dentate gyrus is dependent upon citron- $K$ function, and as there is an apparent loss of dividing cells in postnatal DGZ, we further suggest that citron-K function is required for the production of the SGZ progenitor population from earlier, perhaps fetal, progenitors.

\section{Acknowledgement}

This work was supported by NIH grant MH056524 to J.J.L.

\section{References}

Altman J, Bayer SA (1990a): Mosaic organization of the hippocampal neuroepithelium and the multiple germinal sources of dentate granule cells. J Comp Neurol 301:325-342.

Altman J, Bayer SA (1990b): Migration and distribution of two populations of hippocampal granule cell precursors during the perinatal and postnatal periods. J Comp Neurol 301: 365-381.

Altman J, Das GD (1965): Autoradiographic and histological evidence of postnatal hippocampal neurogenesis in rats. J Comp Neurol 124:319-335.

Alvarez-Buylla A, Lim DA (2004): For the long run: maintaining germinal niches in the adult brain. Neuron 41:683-686.

-Bagri A, Gurney T, He X, Zou YR, Littman DR, Tessier-Lavigne M, Pleasure SJ (2002): The chemokine SDF1 regulates migration of dentate granule cells. Development 129:42494260.

Bayer SA (1980): Development of the hippocampal region in the rat. 1 . Neurogenesis examined with ${ }^{3} \mathrm{H}$-thymidine autoradiography. J Comp Neurol 190:87-114.

-Cameron HA, Woolley CS, McEwen BS, Gould E (1993): Differentiation of newly born neurons and glia in the dentate gyrus of the adult rat. Neuroscience 56:337-344.

-Di Cunto F, Imarisio S, Hirsch E, Broccoli V, Bulfone A, Migheli A, Atzori C, Turco E, Triolo R, Dotto GP, Silengo L, Altruda F (2000): Defective neurogenesis in citron kinase knockout mice by altered cytokinesis and massive apoptosis. Neuron 28:115-127.
Doetsch F, Caille I, Lim DA, Garcia-Verdugo JM, Alvarez-Buylla A (1999a): Subventricular zone astrocytes are neural stem cells in the adult mammalian brain. Cell 97:703-716.

Doetsch F, Garcia-Verdugo JM, Alvarez-Buylla A (1999b): Regeneration of a germinal layer in the adult mammalian brain. Proc Natl Acad Sci USA 96:11619-11624.

Eckenhoff MF, Rakic P (1984): Radial organization of the hippocampal dentate gyrus: a Golgi, ultrastructural, and immunocytochemical analysis in the developing rhesus monkey. J Comp Neurol 223:1-21.

Eriksson PS, Perfilieva E, Bjork-Eriksson T, Alborn AM, Nordborg C, Peterson DA, Gage FH (1998): Neurogenesis in the adult human hippocampus. Nat Med 4:1313-1317.

Gage FH, Coates PW, Palmer TD, Kuhn HG, Fisher LJ, Suhonen JO, Peterson DA, Suhr ST, Ray J (1995): Survival and differentiation of adult neuronal progenitor cells transplanted to the adult brain. Proc Natl Acad Sci USA 92:11879-11873.

-Galceran J, Miyashita-Lin EM, Devaney E, Rubenstein JL, Grosschedl R (2000): Hippocampus development and generation of dentate gyrus granule cells is regulated by LEF1. Development 127:469-482.

- Garcia AD, Doan NB, Imura T, Bush TG, Sofroniew MV (2004): GFAP-expressing progenitors are the principal source of constitutive neurogenesis in adult mouse forebrain. Nat Neurosci 7:1233-1241.
Gould E, Reeves AJ, Fallah M, Tanapat P, Gross CG, Fuchs E (1999): Hippocampal neurogenesis in adult Old World primates. Proc Natl Acad Sci USA 96:5263-5267.

Guéneau G, Privat A, Drouet J, Court L (1982): Subgranular zone of the dentate gyrus of young rabbits as a secondary matrix. A highresolution autoradiographic study. Dev Neurosci 5:345-358.

Houser CR (1990): Granule cell dispersion in the dentate gyrus of humans with temporal lobe epilepsy. Brain Res 535:195-204.

Houser CR (1992): Morphological changes in the dentate gyrus in human temporal lobe epilepsy. Epilepsy Res Suppl 7:223-234.

Imura T, Kornblum HI, Sofroniew MV (2003): The predominant neural stem cell isolated from postnatal and adult forebrain but not early embryonic forebrain expresses GFAP. J Neurosci 23:2824-2832.

Kaplan MS, Hinds JW (1977): Neurogenesis in the adult rat: electron microscopic analysis of light radioautographs. Science 197:10921094.

Kornack DR, Rakic P (1999): Continuation of neurogenesis in the hippocampus of the adult macaque monkey. Proc Natl Acad Sci USA 96:5768-5763.

Laywell ED, Rakic P, Kukekov VG, Holland EC, Steindler DA (2000): Identification of a multipotent astrocytic stem cell in the immature and adult mouse brain. Proc Natl Acad Sci USA 97:13883-13888. 
Lee SM, Tole S, Grove E, McMahon AP (2000): A local Wnt-3a signal is required for development of the mammalian hippocampus. Development 127:457-467.

Lendahl U, Zimmerman LB, McKay RD (1990): CNS stem cells express a new class of intermediate filament protein. Cell 60:585-595.

Li G, Pleasure SJ (2005): Morphogenesis of the dentate gyrus: what we are learning from mouse mutants. Dev Neurosci 27:93-99.

-LoTurco JJ, Sarkisian MR, Cosker L, Bai J (2003): Citron kinase is a regulator of mitosis and neurogenic cytokinesis in the neocortical ventricular zone. Cereb Cortex 13:588-591.

Lu M, Grove EA, Miller RJ (2002): Abnormal development of the hippocampal dentate gyrus in mice lacking the CXCR4 chemokine receptor. Proc Natl Acad Sci USA 99:70907095.

Merkle FT, Tramontin AD, García-Verdugo JM, Alvarez-Buylla A (2004): Radial glia give rise to adult neural stem cells in the subventricular zone. Proc Natl Acad Sci USA 101:1752817532 .
Palmer TD, Takahashi J, Gage FH (1997): The adult rat hippocampus contains primordial neural stem cells. Mol Cell Neurosci 8:389404.

Parent JM, Yu TW, Leibowitz RT, Geschwind DH, Sloviter RS, Lowenstein DH (1997): Dentate granule cell neurogenesis is increased by seizures and contributes to aberrant network reorganization in the adult rat hippocampus. J Neurosci 17:3727-3738.

-Rickmann M, Amaral DG, Cowan WM (1987): Organization of radial glial cells during the development of the rat dentate gyrus. J Comp Neurol 264:449-479.

- Roberts MR, Bittman K, Li WW, French R, Mitchell B, LoTurco JJ, D’Mello SR (2000): The flathead mutation causes CNS-specific developmental abnormalities and apoptosis. J Neurosci 20:2295-2306.

Sarkisian MR, Frenkel M, Li W, Oborski JA, LoTurco JJ (2001): Altered interneuron development in the cerebral cortex of the flathead mutant. Cereb Cortex 11:734-743.

Sarkisian MR, Li W, Di Cunto F, D’Mello SR, LoTurco JJ (2002): Citron-kinase, a protein essential to cytokinesis in neuronal progenitors, is deleted in the flathead mutant rat. J Neurosci 22:RC217.
Sarkisian M, Rattan S, D’Mello S, LoTurco J (1999): Characterization of seizures in the flathead rat: a new genetic model of epilepsy in early postnatal development. Epilepsia 40: 394-400.

- Schlessinger AR, Cowan WM, Gottlieb DI (1975): An autoradiographic study of the time of origin and the pattern of granule cell migration in the dentate gyrus of the rat. J Comp Neurol 159:149-175.

Seri B, García-Verdugo JM, Collado-Morente L, McEwen BS, Alvarez-Buylla A (2004): Cell types, lineage, and architecture of the germinal zone in the adult dentate gyrus. J Comp Neurol 478:359-378.

-Seri B, Garcia-Verdugo JM, McEwen BS, Alvarez-Buylla A (2001): Astrocytes give rise to new neurons in the adult mammalian hippocampus. J Neurosci 21:7153-7160.

Zhou CJ, Zhao C, Pleasure SJ (2004): Wnt signaling mutants have decreased dentate granule cell production and radial glial scaffolding abnormalities. J Neurosci 24:121-126. 